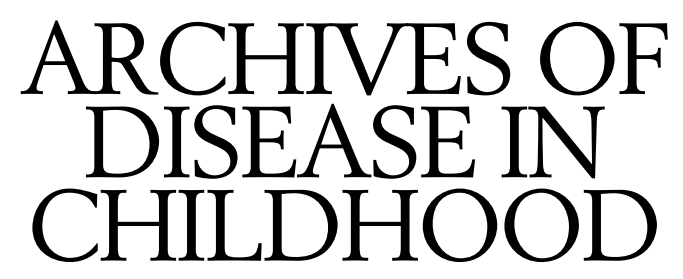

The fournal of the Royal College of Paediatrics and Child Health

\title{
Annotations
}

\section{The vitamin K debacle: cut the Gordian knot but first do no harm}

As clinicians who have contributed to the data and the discussions about vitamin $\mathrm{K}$ prophylaxis to prevent vitamin $\mathrm{K}$ deficiency bleeding (VKDB) in infancy, we are acutely aware that the debate about the advantages and disadvantages of various forms of prophylaxis may undermine public confidence and so put at risk potential health benefit. Echoing Von Kries $^{1}$ we suggest in this annotation that a radical solution is urgently required and could be introduced. Sadly, the history of vitamin $\mathrm{K}$ prophylaxis is replete with examples of medical practice driven by fashion and controversy. For example, over enthusiastic dosing with synthetic vitamin $\mathrm{K}_{2}$ (Synkavit, Roche, Herts, UK) during the 1960s led to complications of haemolysis, hyperbilirubinaemia, and kernicterus, which brought the practice of vitamin K prophylaxis into some disrepute. The most dramatic controversy, still current, originates from reports by Golding and colleagues that intramuscular prophylaxis with vitamin $\mathrm{K}_{1}$ may be associated with an increased incidence of childhood leukaemia and cancer. ${ }^{2}$ These controversies have been extensively reviewed. ${ }^{13}$

\section{Effects of uncertainty and controversy in medical practice}

The potential for poorly informed public debate to generate anxiety and undermine confidence in preventive procedures was well illustrated in the 1980s by the reported association of pertussis immunisation with an encephalopathy of infancy. Loss of public confidence in the immunisation led to mortality and morbidity among children whose parents declined it, ${ }^{45}$ while many professionals felt unable to recommend it for all infants. The risk of a similar situation arising from reports of an association between measles, mumps, and rubella immunisation and infantile autism resulted in prompt and unequivocal advice from the Chief Medical Officer, which supported public confidence, although there was an increase in immunisation refusals. ${ }^{6}$ Another example was the increase in unplanned pregnancies that resulted from the inadequately informed debate about the risks of some oral contraceptive agents. The role of the media early in this episode has been examined. ${ }^{78}$ In each of these examples, properly reported research findings were widely, and sometimes sensationally, debated in the public media, which led to widespread concern. Later contradictory and reassuring evidence was not given the same media priority so that the original findings are still believed by a significant proportion of the public. For reasons that are unclear there is often a lack of confidence in medical and public health opinion, perhaps arising from a suspicion of hidden motives. Public perception of the BSE/nvCJD crisis has contributed to the view that the advice given by government departments and their scientific advisors is sometimes driven by political expediency, only to be later overturned when found unworkable.

\section{Intramuscular vitamin $\mathbf{K}$}

Public confidence in the safety of vitamin K prophylaxis by intramuscular injection has been severely shaken by the reported association with later childhood malignancy. There is now professional confidence that any increased risk of cancer and leukaemia in childhood must be substantially less than the twofold increase suggested by Golding et al; however, there is little prospect that it will be possible to prove in the near future, if ever, that there is no additional risk. ${ }^{3}$ The risk of childhood cancer is estimated to be 1 in $600(166 / 100000)$ live births and the risk of VKDB in "normal risk" infants selected to receive no prophylaxis is in the order of $10 / 1000$ live births. ${ }^{9}$ Even a $10 \%$ increase in the risk of cancer would adversely affect more children than would benefit from the complete prevention of VKDB.

We believe that confidence in prophylaxis by intramuscular injection will be difficult to restore fully and might be further undermined by future research, litigation or withdrawal of Konakion (Roche). Other factors making intramuscular prophylaxis unattractive to parents and professionals include concerns about the "medicalisation" of birth by intramuscular injection and the risks of the injection itself, including the inadvertent administration of the wrong drug. Finally, there is no certainty that a single intramuscular injection of the mixed micellar preparation (Konakion MM, Roche), which may become the only preparation available, will be efficacious throughout the 3-6 month period of risk, as it may not form the muscle depot thought to provide extended absorption. ${ }^{10}$

Offering oral prophylaxis using one of the current UK regimens may present other problems for professionals. The only licensed preparation for oral prophylaxis (Konakion MM) is expensive in terms of drug cost and professional time-supplied only in glass vials it is unsuitable for routine administration by mothers and so professional administration of each dose is specified in the datasheet. After oral administration, the $2 \mathrm{mg}$ licensed 
dose achieves peak vitamin $\mathrm{K}_{1}$ blood concentrations similar to those after standard Konakion $1 \mathrm{mg}$ intramuscular injection. If there is any toxicity from the vitamin itself, each oral dose of the new preparation could be as toxic as the traditional intramuscular prophylaxis. An alternative preparation, Orokay (SmithKline Beecham, Herts, UK), is quite widely used, is easily given by unsupervised mothers, and is cheaper, but presently unlicensed. Finally, providers and professionals must be confident that they can ensure the reliable and timely delivery of multiple doses over a prolonged follow up.

As a result of these difficulties, we have in the UK an extraordinary variety of protocols for vitamin $\mathrm{K}$ prophylaxis. The most recent advice ${ }^{3}$ complicates this further by recommending two oral doses even for low risk, formula fed infants. The most recent study ${ }^{11}$ found that very few units continued a "selective policy" of giving prophylaxis only to babies considered most at risk of VKDB. The vast majority gave vitamin $\mathrm{K}$, in some form, to all newborns but there were numerous permutations of preparation used, route of administration, dose, and number of doses.

A recent letter to all doctors from the Chief Medical and Nursing Officers addresses the issue. ${ }^{3}$ Four questions are considered but only two of them can be answered satisfactorily. The question, "Do all babies need additional vitamin $K$ ?" is answered with a firm recommendation that all babies should be offered prophylaxis to prevent the 4-6 deaths and 10-20 cases of brain damage that would occur each year in the UK if vitamin $\mathrm{K}$ was given selectively only to infants perceived to be at high risk. The question, "Which babies are at greater risk of bleeding?" is answered by describing those whose increased risk of VKDB can be identified at birth and by emphasising the later importance of prolonged jaundice and warning bleeds.

It is not possible, however, to give unequivocal answers to either of the two critical questions. The answer to, "Could vitamin K be harmful?" concludes that "the available data do not support an increased risk of cancer, including leukaemia, caused by vitamin K" but adds that "it is not possible to exclude a small increased risk in leukaemia due to limitations of the data". We believe that because it is impossible to give unequivocal reassurance on this point it is also impossible to make a firm recommendation of a single regimen acceptable to all. Thus the last question, "How can vitamin $\mathrm{K}$ be given?" is answered by offering several regimens, none of which is clearly preferred or recommended, re-emphasising the responsibilities of providers and parents to make decisions and to record the consent, prescription, and administration of medicines whether licensed or unlicensed. Similar conclusions were drawn by Logan and Gilbert in a structured review. ${ }^{12}$

While it is understandable that none of these regimens could be recommended in the expectation that it would receive universal support, we believe that continuing the present uncertainty will further undermine public confidence in vitamin $\mathrm{K}$ prophylaxis and possibly in other areas of preventive medicine. The dangers are illustrated by the response of some midwives to this problem; at the 1997 annual general meeting of the Royal Institute of Midwives it was debated whether vitamin $\mathrm{K}$ prophylaxis should be abandoned altogether.

\section{A radical solution: cut the Gordian knot}

Von Kries et al likened the vitamin $\mathrm{K}$ dilemma to the problem posed by the apparently untieable Gordian knot. ${ }^{1}$ Perhaps our colleagues in the Netherlands were inspired by Alexander the Great's decisive, albeit unsporting solu- tion (he cut the knot with his sword), when they formulated their radical and equally logical prophylaxis regimen. As bottle fed babies receive sufficient vitamin $\mathrm{K}$ in their supplemented formulae to prevent VKDB (with rare exceptions, even those with liver disease are protected), it is logical to give breast fed babies an equivalent supplement of vitamin $\mathrm{K}_{1} 25 \mu \mathrm{g}$ daily. The tiny dose avoids the grossly unphysiological peaks of plasma vitamin produced by other regimens, while daily dosing should circumvent the problem of infants' very limited ability to store the vitamin. (It has been suggested that intramuscular Konakion $1 \mathrm{mg}$ gives reliable prophylaxis for many weeks because a supplementary "store" is formed at the injection site. ${ }^{10}$ ) Since 1992 the following regimen has been used successfully in the Netherlands: normal risk infants receive vitamin $\mathrm{K}_{1} 1 \mathrm{mg}$ orally at birth (given intramuscularly to those with high risk features) and, from 7 days until 3 months of age, breast fed infants are given vitamin $K_{1} 25 \mu \mathrm{g}$ daily as oral drops by their mothers. The drops are sufficiently dilute to be considered as a food additive and so do not require a drug licence.

While it would be hard to prove that daily administration of vitamin $K_{1}$ in this way has no adverse effects, it is acceptable on the basis that it has been practised in formula feeding for many years.

Clearly efficacy would depend on parents giving the drops reliably; the Dutch experience suggests that this is unlikely to be a major problem ${ }^{13}$ as does the Danish experience with a regimen of multiple, parent administered doses. ${ }^{14}$ Breast feeding mothers tend to be especially motivated to provide optimum care for their infants; we believe that they would be happy to take on the responsibility of giving the drops and would feel it appropriate to do so. Even if as many as $10 \%$ of breast feeding mothers failed to give any prophylaxis the prevalence of $\mathrm{VKDB}$ would be expected to fall to less than $1 / 100000$ live births. Other advantages would be the clear transfer of responsibility to parents for the well being of their infants and, perhaps, more importantly, removal of the possibility that medical intervention may be harming some infants, a fear, which however ill founded, is of extreme importance to parents and providers.

A preparation providing $25 \mu \mathrm{g}$ phytomenadione (vitamin $\mathrm{K}_{1}$ ) per day in a drop formulation is already marketed in the Netherlands. This is formulated in arachis oil, a solvent no longer favoured in the UK because of peanut allergy, while Orokay is formulated in coconut oil. Such a preparation could be developed as a food additive for breast fed infants and should not require licensing under the Medicines Act. While there is currently debate about the licensing of vitamins, and we understand that a working party is to be set up by the Ministry of Agriculture Food and Fisheries and the Department of Health to examine the question, we do not believe this should prevent immediate action.

Breast feeding mothers are already advised to provide supplements of vitamins A, B group, C, and D for their infants from the age of 2 months. These vitamins, together with vitamin $\mathrm{K}$, are added to infant formulae. Breast feeding mothers can therefore be reassured that breast milk has many advantages over formula feeds and, supplemented by vitamin $\mathrm{K}$ and $\mathrm{ABDC}$ drops, would have no known disadvantages compared to even the most modern infant formulae. This would be a sound basis on which to mount a publicity campaign to promote both vitamin $\mathrm{K}$ prophylaxis and breast feeding.

The vitamin $\mathrm{K}$ muddle has already gone on for too long. Let us bring it to an end by developing a daily drop preparation and adopting, for all normal risk breast fed infants, the regimen that has served so 
well in the Netherlands. Rates of VKDB should be lower than at present and any possibility, however remote, of prophylaxis harming some normal risk infants will be removed.

J H TRIPP

Senior Lecturer in Child Health,

A W MCNINCH

Consultant Paediatrican,

Department of Child Health,

Royal Devon and Exeter Hospital,

Exeter EX2 5DW, UK

1 Von Kries R. Neonatal vitamin K prophylaxis: the Gordian knot still awaits untying. BMF 1998;316:161-2.

2 Golding J, Paterson M, Kinlen LJ. Factors associated with childhood cancer in a national cohort study. Br f Cancer 1990;62:304-8.

3 Vitamin K for newborn babies. Department of Health Publications 5 May 1998; PL/CMO/98/3; PL/CNO/98/4.

4 Nicoll A, Gardener A. Unrecognised post perinatal mortality due to whooping cough. Arch Dis Child 1988;63:41-8.

5 Nicoll A, Elliman D, Ross E. MMR vaccination and autism 1998; deja vu-pertussis and brain damage 1994? BMf 1998;316:715-16.
6 Begg N, Ramsay M, White J, Bozoky Z. Media dents confidence in MMR vaccine. BMF 1998;316:561.

7 Wetherall M. The "pill scare" of October 1995, the media and the public understanding of science. British fournal of Family Planning 1996;22:1513

8 Allison C, Roizen J, Olivier P. The 1995 pill scare: women's perceptions of risk and sources of information. British fournal of Family Planning 1997;23: 79-82.

9 McNinch AW,Tripp JH. Haemorrhagic disease of the newborn in the British Isles: two year prospective study. BMF 1991;303:1105-9.

10 Loughnan P, McDougall P. The duration of vitamin K efficacy: does intramuscular vitamin $\mathrm{K}$ act as an unintended depot preparation? f Paediatr Child Health 1996;32:251-4.

11 Barton JS, Tripp JH, McNinch AW. Neonatal vitamin K prophylaxis in the British Isles: current practice and trends. BMF 1995;310:632-3.

12 Logan S, Gilbert R. Vitamin K prophylaxis for haemorrhagic disease of the newborn: an appraisal of the effectiveness of oral regimens. London: Institute of Child Health, 1998.

13 Cornelissen M, von Kries R, Loughnan P, Schubiger G. Prevention of vitamin $\mathrm{K}$ deficiency bleeding: efficacy of different multiple oral dose schedules of vitamin K. Eur ₹ Paediatr 1997;156:126-30.

14 Norgaard Hansen K, Ebbesen R. Neonatal vitamin K prophylaxis in Denmark: three years' experience with oral administration during the first threemonths of life compared with one oral administration at birth. Acta Paediatr 1996;85:1137-9.

\section{Inferences for health provision from survival data in cystic fibrosis}

Patients with cystic fibrosis (CF), their families, carers, insurers, health care planners, and CF carriers all have an interest in knowing the lifespan of people with the disease. Evidence-based medicine is now explicitly practised by many clinicians in their everyday clinical work. This practice should include prognosis, ${ }^{1}$ where the expected lifespan is the most important statistic.

However, clinicians with a responsibility for these patients are faced with a large literature on the survival of people with $\mathrm{CF}$, which presents a contradictory picture. My purpose is to show how these contradictions may be resolved by reference to other published material. I have examined three "notable" observations to show what inferences may be reasonably drawn from them concerning the lifespan of people with CF.

In the absence of properly conducted randomised controlled clinical trials, observational methods have been used to try to determine the relative efficacy of different models of providing clinical care, even though such studies provide only weak evidence. ${ }^{2}$ In particular, the possible advantage of care at specialist centres compared to care by local paediatricians has been debated for many years. Because of the relatively small numbers of cases and local variations in the care delivered, international comparisons have been used to assess these two different strategies for care.

\section{Basic epidemiological considerations}

All data should relate to a well defined population, preferably the residents of a geographical region. Where a group is studied (such as people with CF) rules should exist which allow individuals to be allocated to that group (diagnosed) and members should be found by population screening. Case finding is less reliable than screening.

\section{Two different ways of estimating survival}

There are two different methods for calculating survival. Cohort survival would identify all people born with CF in a given time period; each subsequent year those surviving would be noted. Eventually all the cohort will have died and a complete picture of their survival will be available. This method takes a very long time. Estimates of median survival derived from such data are unlikely to be relevant to newly born cases because of improvements in treatment leading to a longer lifespan.

An alternative is the current survival method, which only requires observations over one year. All current cases alive in one year have to be identified and the deaths in that year noted. For each age, a mortality rate is calculated. It is then assumed that the calculated age specific mortality rates will apply to the current cohort over their future lifespan. Applying these mortality rates to the current cohort gives an estimate of their future survival. These data are the most up to date available, and represent a useful summary of the current age specific mortality rates. A fuller explanation of survival calculations is obtainable from many medical statistics textbooks. ${ }^{3}$ Caution is required when using such data to predict future survival, particularly for the CF population which has seen regular improvements in survival for the past 30 years.

EXAMPLE 1: AN EARLY ESTIMATE OF SURVIVAL

Over 30 years ago, life table data were given for children with $\mathrm{CF}$ attending one large hospital. ${ }^{4}$ This early quantitative data suggested that only a quarter survived to age 16 years for the period 1943-64. These data present such a striking contrast to normal population survival that any methodological weaknesses in the study cannot account for this difference.

There was little reported improvement in survival between those born in the first half of the period and those in the second. Although not stated explicitly, the results must have been obtained by current survival methods. Thus, this lack of improvement could be caused by survivor bias in the earlier group, with survivors being milder cases. The one year survival was given as over $80 \%$, which is equivalent to that reported for the $1968-70$ UK cohort. ${ }^{5}$ The survival to later ages in the 1968-70 cohort was much better than that of the 1943-64 group. Historical data for the UK show a continuing improvement in the mortality 
rate for all ages. ${ }^{5}$ This apparent lack of improvement in the survival of these infants compared to the improvement in all the other ages is consistent with an overestimate of the infant survival in the first group. Clinic based data only have cases that have survived long enough to be referred to the clinic. This effect can be seen in the relatively low mortality rates quoted for the first year of life in clinic based data compared to population based data. There is a smaller bias, in the opposite direction, caused by late diagnosis of adults (which still occurs) which would not be included in the data from a paediatric clinic. This early work correctly highlighted the survival consequences of $\mathrm{CF}$. The weak methodology militates against using these results as a basis for measuring improvements in treatment. However, the survival of UK children with CF during 1943-64 was almost certainly worse than that reported.

EXAMPLE 2: AN INTERNATIONAL COMPARISON OF SURVIVAL TO EVALUATE PATIENT MANAGEMENT

A "comparison of the estimated (current) survival curves for CF in England and Wales, and Victoria, Australia for the years 1976-80" suggested Victoria was $18 \%$ better at $80 \%$ survival to age 20 years (fig 1 ). ${ }^{6}$ After eliminating respiratory infections and sudden infant deaths as possible explanations, attention is drawn to the fact that care for $90 \%$ of the children and adolescents in Victoria was provided by "a specialist centre" which was thought to be essential for optimum care. ${ }^{7}$ This care was contrasted with England and Wales where most children were looked after by their local paediatrician. The paper finished by suggesting the "need for further research in England and Wales into the reasons why the death rate... seems to be substantially higher than in Victoria".

An independent estimate of UK current survival for the period $1980-85$ gives only $55 \%$ survival to age $20 .^{8}$

The current survival estimate for the period 1991-95 to age 20 for Victoria is $56 \%$ although a calculation which removed potential bias in the first few years of life gives $60 \% .^{9}$ The comparable UK figures are $64 \%$ for $1986-87,{ }^{10}$ and $74 \%$ for $1994 .^{5}$

These data could be taken to show that Victoria has seen survival to age 20 fall in 15 years from $80 \%$ to $56 \%$. The UK, which still has $36 \%$ of its patients not attending a specialist centre, ${ }^{11}$ has seen survival to age 20 rise in 10 years from $55 \%$ to nearly $75 \%$. These data span a period when many new therapies were becoming

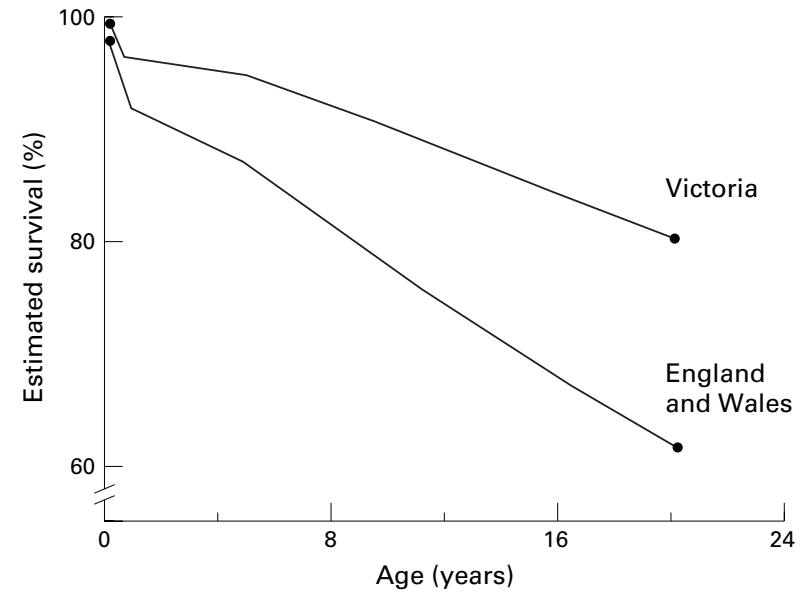

Figure 1 Estimated survival curves for CF in England and Wales, and Victoria, Australia derived from mortality data for the years 1976-80. (Reproduced from Arch Dis Child 1984;59:71-83 with permission of BMF Publishing Group.) available for CF, and CF survival was universally regarded as improving.

These data, in total, present an implausible picture. Victoria is similar to the UK, with clinicians and other health care providers trained to the same exacting standards and a constant exchange of staff between the countries. The most likely explanation is bias in the early Victoria data. The number of deaths were small (26), so small fluctuations in the numbers dying could have a large influence on the data. The death data in the original paper, showing $20 \%$ mortality to age 20 years in Victoria, was most likely an underestimate of the longer term trend. Publication bias could be a contributory factor; the paper might not have been published had the results shown the opposite effect.

Contrary to the original claim, ${ }^{6}$ these data provide no evidence that the "specialist centre" of Victoria confers a better survival than the less centralised service provided in the UK.

EXAMPLE 3: NO RECENT IMPROVEMENTS IN SURVIVAL IN SOME COUNTRIES

The estimated median survival for the Canadian population has been falling since $1989,{ }^{12}$ while the data from USA show levelling off, ${ }^{13}$ with the estimates for median survival being about 30 years.

However, data from Denmark show spectacular results with $80.4 \%$ survival to 50 years. ${ }^{14}$ The combination of these three sets of results has prompted the need for "a thoughtful review of current treatment programs and how they may be altered to improve the outlook for CF patients". ${ }^{15}$

Both the latest Canadian and USA current survival curves show the same convex shape as the UK data (fig 2). This convexity is caused by successive cohorts having better survival than the previous. If the cohorts from North America are showing similar patterns to the UK data, then improving survival must be inevitable for the next few years at least. This is confirmed by the best estimates that can be made of the annual CF births and deaths. For both countries, births per year are consistently about double the deaths. This can only happen when survival is increasing, there being no suggestion of any change in the incidence of the disease. The apparent ending of improvement in survival is a combination of chance fluctuations in the numbers dying and probably some short term corrections for overestimated survival in previous years because of the weak definitions of the populations under study.

The Danish data are just not plausible. The survival curve estimates no deaths for people with CF between the ages of 35 and 50 years of age! These estimates are very

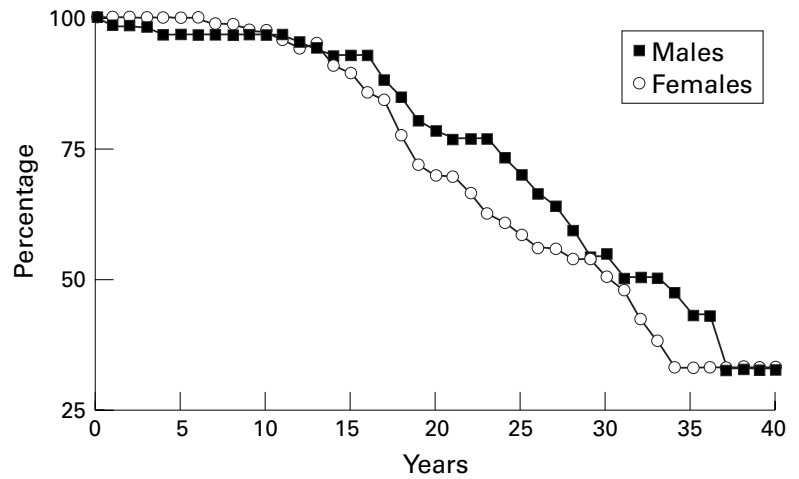

Figure 2 Current survival of UK residents with CF for 1994 for both sexes. (Reproduced from Arch Dis Child 1997;77:493-6 with permission of BMF Publishing Group.) 
imprecise, being based on 270 patients and on what appear to be four deaths. Before there is a need for "a thoughtful review of current treatment programs..." based on these data, it would be better if the data were thoughtfully reviewed.

In large populations, there is no evidence that one country with a well developed knowledge and treatment program for $\mathrm{CF}$ has a markedly better survival than any other similar country, and thus no evidence that one country's method of delivering health care to people with CF is "the best".

The perplexing issue of the expected survival of a newborn baby with CF remains unresolved as there are insufficient data to make a reliable estimate. Recent cohort data $^{5}$ record dramatic improvements in survival over the last 30 years. Attempts at extrapolating these improvements to predict survival over the next 50 years or more are fraught with uncertainty. The latest cohort data from the UK show that those born in the 1990s have a survival which is close to that of the overall population. For CF cases born in 1995 (about 300) there have been two deaths reported in the first year of life. At best, the survival of CF cases might track that of the base population. At worst, there might be some unexpected complication, which, in the future, will halt these improvements resulting in a median survival below 30 years.

\section{Conclusions}

A guess as to the expected lifespan by a supposedly reputable source, based on an analysis with questionable assumptions, will almost certainly be wrong and risks disadvantaging one group or another. Insurers, health care planners, patients, their families, and CF carriers all have an interest in knowing the lifespan. It is better to acknowledge that this is unknown, and plan with that uncertainty, rather than take decisions on incorrect data.

P A LEWIS

Public Health Group,

School of Postgraduate Medicine,

University of Bath,

Bath BA2 7AY, UK

1 Sackett DL, Richardson WS, Rosenburg W, Haynes RB. Evidence based medicine. New York: Churchill Livingstone, 1997.

2 McKinnell I, Elliott J. The Cochrane electronic library. Anglia and Oxford: NHS Executive, 1997.

Hill AB. A short textbook of medical statistics. London: Hodder and Stoughton, 1980.

4 Mantle DJ, Norman AP. Life-table for cystic fibrosis. BMf 1966;ii:1238-41.

5 Dodge JA, Morison S, Lewis PA, et al. Incidence, population, and survival of cystic fibrosis in the UK, 1968-95. Arch Dis Child 1997;77:493-6.

6 Phelan P, Hey F. Cystic fibrosis in England and Wales and in Victoria, Australia 1976-1980. Arch Dis Child 1984;59:71-83.

7 Stern RC, Boat TF, Doershuk CF, et al. Course of cystic fibrosis in 95 Stern RC, Boat TF, Doershuk CF,
patients. F Paediatr 1976;89:406-11.

8 Dodge JA, Goodall J, Geddes D, et al. Cystic fibrosis in the United Kingdom 1977-85: an improving picture. BMF 1988;297:1599-602.

9 Australian Cystic Fibrosis Associations Federation. Annual data report 1995/6. North Ryde, New South Wales: Australian Cystic Fibrosis Associations Federation, 1996.

10 Dodge JA, Morison S, Lewis PA, et al. Cystic fibrosis in the United Kingdom, 1968-1988: incidence, population and survival. Pediatr Perinat Epidemiol 1993; 7:157-66

11 Morison S, Dodge JA, Cole TJ, et al. Height and weight in cystic fibrosis: a cross sectional study. Arch Dis Child 1997;77:497-500.

12 Canadian Patient Data Registry. National report 1994. Toronto, Ontario: Canadian Cystic Fibrosis Foundation, 1995.

13 Cystic Fibrosis Foundation Patient Registry. 1995 annual data report. Bethesda, Maryland: Cystic Fibrosis Foundation, 1996.

14 Frederiksen B, Lanng S, Koch C, Høiby N. Improved survival in the Danish center-treated cystic fibrosis patients: results of aggressive treatment. Pediatric Pulmonology 1996;21:153-8.

15 Corey M. Survival estimates in cystic fibrosis: snapshot of a moving target. Pediatric Pulmonology 1996;21:149-50. 